\title{
Hypoxia-inducible factor-1 $\alpha$ regulates energy fuel metabolism and mediates metabolic plasticity during ammonia stress response in ovarian cancer stem- like cells
}

\author{
Shojiro Kitajima ${ }^{1,5}$, Kian Leong Lee ${ }^{2}$, Hiroki Hikasa ${ }^{3}$, Shinji Matsunaga ${ }^{1}$, Takehiro Yamaguchi $^{1}$, \\ Shuhei Tomita ${ }^{1}$, Marito Araki $^{4}$, Hiroyuki Kato ${ }^{5}$, Lorenz Poellinger ${ }^{5}$ \\ ${ }^{1}$ Pharmacology, Osaka City University Graduate School of Medicine, Japan, ${ }^{2}$ Cancer \& Stem Cell Biology Program, \\ Duke-NUS Medical School, Singapore, ${ }^{3}$ Department of Biochemistry, School of Medicine, The University of \\ Occupational and Environmental Health, Japan, ${ }^{4}$ Department of Transfusion Medicine and Stem Cell Regulation, \\ Juntendo University Graduate School of Medicine, Japan, ${ }^{5}$ Cancer Science Institute of Singapore, National University \\ of Singapore, Singapore
}

Ammonia is produced by cancer cells as a by-product of glutamine metabolism during rapid cell growth. Although the toxicity of ammonia has been well known, specific factors that deal with ammonia-induced cellular stress and regulate cell metabolism to survive have yet to be identified. We demonstrated that the hypoxia-inducible factor-1 $\alpha$ (HIF-1 $\alpha)$ is stabilised and activated during ammonia stress response. The ablation of HIF-1 $\alpha$ promoted apoptosis caused by high levels of ammonia. Moreover, we identified glutamine synthetase (GS) as a key driver of cancer cell proliferation under ammonia stress and glutamine-dependent metabolism in ovarian cancer stem-like cells marked by the expression of CD90. Interestingly, activated HIF-1 $\alpha$ counteracts GS function in glutamine metabolism by facilitating glycolysis leading to elevated glucose dependency. Our studies reveal the hitherto unknown functions of HIF-1 $\alpha$ that promote metabolic plasticity during ammonia stress response in the cancer stem-like cells where GS facilitates cell proliferation and HIF-1 $\alpha$ contributes to remodeling in energy fuel usage resulting in attenuated proliferation but conversely promoting cell survival. 